\title{
LES ORIENTATIONS POUR LES JEUNES : ETHOS, SCÉNOGRAPHIE ET STÉRÉOTYPES ${ }^{1}$
}

\author{
ANNA FLORA BRUNELLI ${ }^{2}$ \\ (UNESP - Campus São José do Rio Preto) \\ MARCELA FRANCO FOSSEY ${ }^{3}$ \\ (Postdoctoral - UNICAMP) \\ MARILIA MOLINA FURLAN ${ }^{4}$ \\ (Doctorat UNESP - Campus São José do Rio Preto)
}

\begin{abstract}
RESUMO: Neste trabalho, com base nas reflexões que Dominique Maingueneau desenvolve sobre os aspectos enunciativos do discurso, analisamos o discurso de orientação para adolescentes, procurando apreender a imagem de adolescente que o discurso projeta. Mais exatamente, considerando certos mecanismos enunciativos que caracterizam esse discurso, analisamos o ethos do enunciador e a cenografia em que emerge. A análise revela que o fiador, inserido em cenografias de uma conversa informal, está relacionado a um ethos juvenil. Além disso, a análise revela que, nesse discurso, a imagem do enunciatário, considerado essencialmente como um jovem inseguro e imaturo, prevalece sobre a do enunciador.
\end{abstract}

Palavras-chave: orientações; adolescentes; ethos.

ABSTRACT: In this paper, based on the thoughts that Dominique Maingueneau develops on the enunciative aspects of discourse, we analyze the orientation discourse for teenagers, trying to apprehend the image of teenagers that the discourse projects. More precisely, considering certain enunciative mechanisms that characterize this discourse, we analyze the ethos of the enunciator and the scenography in which it emerges. The analysis reveals that the guarantor, inserted in scenarios of an informal conversation, is related to a juvenile ethos. Moreover, the analysis reveals that in this discourse the image of the enunciator, considered essentially as an insecure and immature young person, prevails over that of the enunciator.

Keywords: guidances; teenagers; ethos.

${ }^{1}$ Les données analysées dans cet article correspondent au recoupement des résultats de deux recherches: Fossey (2011; FAPESP: 2008/53363-5) et Furlan (2013).

${ }^{2}$ FAPESP (2014/21825-0); email: anna@ibilce.unesp.br

${ }^{3}$ email: marcela.ff@gmail.com

${ }^{4}$ CAPES (33004153069P-5); email: mariliamolinafurlan@yahoo.com.br 


\section{INTRODUCTION}

À partir des réflexions de Maingueneau sur l'énonciation et des notions qu'il mobilise pour cela, nous analysons dans ce travail quelques textes contemporains adressés au public adolescent brésilien pour révéler les mécanismes énonciatifs qui caractérisent et/ou spécifient le discours d'orientation destiné à ce public, tout en cherchant à appréhender le stéréotype d'adolescent qui en émerge.

Notre objectif est d'apporter une contribution aux réflexions sur le rôle des stéréotypes dans les discours. Dans le cadre de la psychologie sociale, les stéréotypes sont, en général, des manières de connaître les individus, pris collectivement et caractérisés par une fausse représentation (cf. AMOSSY et PIERROT, 2001). Ce sont essentiellement des représentations cristallisées d'un groupe social à partir desquelles on peut non seulement comprendre le réel, mais aussi et surtout, le catégoriser et agir sur lui. En tant que produits culturels préexistants, les stéréotypes sont un thème qui mobilise fructueusement les présupposés théoriques et méthodologiques de l'Analyse du Discours, mettant en évidence le rôle de la mémoire discursive dans la constitution des discours. En outre, les travaux de la psychologie sociale révèlent que les stéréotypes très répandus deviennent culturellement plus accessibles, et de la sorte ils peuvent affecter le comportement des gens même quand ceux-ci ne les endossent pas, ce qui renforce notre intérêt sur la façon dont les discours collaborent à leur diffusion.

Dans la première partie du travail, on présente le cadre théorique et méthodologique sur lequel on se base pour développer cette analyse. Comme nous l'avons déjà dit, il s'agit des réflexions de Maingueneau sur l'énonciation, en mettant l'accent sur les notions d'ethos et de scénographie. ${ }^{5}$ Ensuite, en explorant des aspects spécifiques de la surface discursive, nous analysons l'ethos du garant du discours d'orientation pour les adolescents, en cherchant à appréhender l'image d'adolescent projetée par le discours en question. De cette façon, nous pouvons dire que nous développons dans ce travail une analyse linguistique de ce discours, ce que nous considérons comme une manière privilégiée d'appréhender la discursivité car, comme on le sait, bien que la discursivité ait un ordre qui lui est propre e qui ne se confond pas avec l'ordre de la langue, c'est dans la langue que cet ordre se réalise.

Les textes sélectionnés pour cette analyse sont destinés à des adolescents et, quoiqu'ils divergent dans leur genre, ils sont proches dans la mesure où il s'agit de productions contemporaines destinées à donner un type d'orientation aux adolescents ; de sorte que ce qui les rapproche est le fait qu'ils appartiennent au même genre de discours, ainsi défini par le critère de finalité sociale. ${ }^{6}$

\footnotetext{
${ }^{5}$ Cf. Maingueneau (2006, 2008).

${ }^{6} \mathrm{Cf}$. Maingueneau (2015).
} 


\section{LES RÉFLEXIONS DE MAINGUENEAU SUR L'ÉNONCIATION: LES NOTIONS D'ETHOS ET DE SCÉNOGRAPHIE}

D'un point de vue général, on peut dire que la notion d'ethos concerne l'image que l'énonciateur du discours projette de lui-même par son propre discours, c'est-à-dire par la façon dont il s'exprime, par les caractéristiques de son énonciation. Dans ces termes, l'ethos a trait à la manière de s'exprimer de l'énonciateur, ce qui ne se confond pas avec ce que l'énonciateur dit de luimême, même si cette information a aussi son importance pour appréhender l'image qu'il projette. ${ }^{7}$

Dans le contexte théorique spécifique dans lequel Maingueneau travaille, la notion d'ethos discursif se configure comme une voix qui se révèle dans l'énonciation, qui la soutient et la légitime, et qui ne se restreint pas aux énoncés verbaux, vu qu'elle fait partie de l'identité de tout discours (Maingueneau, 2008). Outre la dimension vocale qu'elle mobilise, la notion d'ethos concerne aussi un caractère et une corporalité qui correspondent respectivement à des traits psychologiques et à une complexion corporelle.

Dans leur ensemble, ces trois aspects de l'ethos (voix, ${ }^{8}$ caractère et corporalité) projettent l'image de celui qui serait le responsable du texte. Maingueneau appelle cette instance subjective garant, et souligne qu'elle ne se laisse pas percevoir dans le discours seulement comme un statut, mais comme une " voix associée à la représentation d'un corps énonçant historiquement spécifié » (MAINGUENEAU, 2006: 271). Ce corps énonçant correspond à son tour aux représentations sociales qui sont évaluées positivement ou négativement, soit aux stéréotypes que l'énonciation contribue à renforcer et/ou transformer.

Comme l'ethos concerne une image identifiable et qui se distingue par une manière singulière d'expression, Maingueneau observe qu'on peut l'appréhender à partir d'indices très variés, qui vont du registre de la langue et des mots jusqu'à la planification textuelle, en passant par le rythme et la modulation (cf. MAINGUENEAU, 2008: 16).

Outre les propriétés citées, l'ethos est lié à la scène d'énonciation. Dans le cadre des réflexions qu'il développe sur l'énonciation, Maingueneau (2006) emploie la notion de " scène d'énonciation » à la place des notions traditionnelles de « contexte » ou de « situation de communication », qui sont plus proches d'une conception sociologiste de l'énonciation, selon laquelle les interlocuteurs jouent dans le discours des rôles sociaux préalablement définis. D'après cette conception, la situation de communication est essentiellement un cadre, une toile de fond préalable. La notion de « scène » concerne elle aussi les rôles de l'énonciateur et de l'énonciataire qui sont impliqués dans les genres du discours, mais elle va au-delà en

${ }^{7}$ Cf. la notion d'ethos dit (Maingueneau, 2006). Cette notion concerne les passages où l'énonciateur fait référence directe ou indirecte à lui-même ou à sa façon d'énoncer (« je suis un homme du peuple », « je vous parle en tant qu'une personne expérimentée »).

${ }^{8}$ Cette voix, Maingueneau préfère l'appeler ton, dans la mesure où il est possible de parler du « ton » d'un texte de la même manière que l'on parle de celui d'une personne (MAINGUENEAU, 1989: 46). 
mettant en évidence le fait que l'énonciation ne se produit pas seulement par rapport à un espace préalablement institué, défini par le genre du discours: elle instaure aussi un espace spécifique qui est mis en scène par le discours.

Pour Maingueneau (2006), la scène d'énonciation d'un discours est composée de trois scènes: la scène englobante, la scène générique et la scénographie. La scène englobante concerne le type de discours, correspondant à son statut pragmatique. Cette scène est associée au mode de fonctionnement social du discours (par exemple, discours littéraire, discours religieux, discours philosophique, discours publicitaire). La scène générique, à son tour, se rapporte au contrat associé à un genre déterminé de discours (article scientifique, article d'opinion, éditorial journalistique, ordonnance médicale).

Comme le fait observer Maingueneau (2006), la scène d'énonciation peut se limiter à ces deux scènes, avec une mise en relief de la scène générique. Mais le discours peut instituer aussi une autre scène qui renvoie les précédentes au second plan. Dans ce cas, une nouvelle scène est offerte à l'énonciataire comme si le discours y trouvait ses origines, mais cette scène, en réalité, est une scène construite par le discours lui-même, une scène engendrée par lui. La scénographie est, donc, la scène construite par les éléments présents dans le texte. Autrement dit: les textes sont constitués d'éléments (lexique, structures syntaxiques typiques, thèmes, images associées, etc.) desquels découle leur scénographie. Et la scénographie est justement le lieu où le garant du discours s'insère, assumant un certain mode d'énonciation.

Bien que la scénographie soit la scène que le discours présuppose pour qu'il puisse être énoncé, comme nous l'avons déjà dit, le discours doit, à son tour, la valider dans sa propre énonciation pour devenir légitime. Cela signifie que la scénographie n'est pas un « espace » indépendant du discours, comme si le discours pouvait réellement se produire dans un espace déjà construit, mais c'est essentiellement la scène que l'énonciation instaure progressivement comme son propre dispositif de parole, c'est-à-dire la scène que le discours valide progressivement au moyen de sa propre énonciation. Ainsi, la scénographie, avec l'ethos auquel elle est associée, implique, d'après Maingueneau, un processus d'entrecroisement paradoxal:

dès son émergence la parole est portée par un certain ethos, lequel, en fait, se valide progressivement à travers cette énonciation même. La scénographie est ainsi à la fois ce dont vient le discours et ce qu'engendre ce discours; elle légitime un énoncé qui, en retour, doit la légitimer, doit établir que cette scène dont vient la parole est précisément la scène requise pour énoncer dans telle circonstance (MAINGUENEAU, 2006: 68).

À partir de ce qui a été exposé, nous analyserons dans ce qui suit l'ethos du discours d'orientation pour les adolescents. Plus exactement, dans chaque texte sélectionné pour composer le corpus de notre travail, nous nous emploierons à identifier l'ethos du garant du discours et la scénographie d'où il émerge. En fonction des résultats de cette analyse, développée à partir de divers indices de la surface discursive, nous nous efforcerons d'identifier la/les image(s) d'adolescent(s) présente(s) dans ce discours, ce que nous ferons en considérant non seulement le profil du garant, mais aussi l'image de l'énonciataire à qui il s'adresse. 


\section{LES ORIENTATIONS DE SANTÉ ET DE COMPORTEMENT POUR LE JEUNE MANQUANT D'ASSURANCE}

Le premier texte analysé est le livre Tipo assim: adolescente, ${ }^{9}$ écrit par Sonia Francine, ex-présentatrice de MTV Brésil, et le docteur Jairo Bouer, médecin, éducateur et présentateur de télévision qui travaille depuis 20 ans dans le domaine de la communication et qui est actuellement une référence majeure dans les médias brésiliens en ce qui concerne la santé et le comportement des jeunes. Cet ouvrage, publié en 2005 par la maison d'édition Papirus, se présente sous la forme d'un dialogue entre les deux auteurs, qui abordent certains sujets communément rapportés à l'adolescence, comme les drogues, le sexe, la relation avec les parents, l'adaptation et la relation entre amis et avec les groupes, etc., et qui commentent certaines expériences qu'ils ont eues pendant cette phase de leur vie. Il s'agit donc d'un ouvrage orienté vers les adolescents et conçu par des auteurs connus de ce public. Il ne s'agit pas de conseils donnés aux parents pour traiter les situations problématiques de leurs enfants, mais d'une discussion adressée aux adolescents qui cherchent à comprendre le moment qu'ils vivent.

Pour analyser l'ethos des énonciateurs, nous observerons de façon précise la matérialité linguistique du texte à partir de trois éléments d'investigation, à savoir: les occurrences des marqueurs discursifs (2.1), les occurrences des éléments modaux (2.2) et les aspects du lexique employé (2.3).

\subsection{Les marqueurs discursifs}

D'une manière générale, les marqueurs discursifs (MD, désormais) assurent l'ancrage pragmatique de l'énoncé en manifestant la présence des énonciateurs dans l'énoncé et en révélant certaines caractéristiques du processus énonciatif. Plus exactement, les MD sont comme des embrayeurs des énoncés avec leurs conditions d'énonciation, renvoyant aux instances de production du discours et définissant la relation de ces instances avec la structure textuelle-interactive. Ainsi, les MD concernent

les articulations textuelles et les relations interpersonnelles, avec une mise en relief fonctionnelle dans l'un ou l'autre de ces aspects, qui particularisent deux grands sous-ensembles: les MD fondamentalement séquenceurs et ceux fondamentalement interactifs. Les particularités fonctionnelles différenciées (...) ne sont pas exclusives ni s'excluent l'une à l'autre; au contraire, elles sont en relation avec des degrés corrélatifs de projection des deux fonctions de base: le plus grand poids du facteur interactionnel correspond normalement à une dilution du rôle articulateur; et inversement, la croissance de l'action séquenciatrice côtoie un degré plus atténué de manifestation du jeu des relations interpersonnelles (OLIVEIRA E SILVA, RISSO et URBANO, 2006: 424-5) (notre traduction).

\footnotetext{
${ }^{9}$ Il serait assez difficile de trouver un équivalent en français pour l'expression brésilienne « tipo assim ». On pourrait la comparer plus ou moins à l'expression « kind of » (ou « kinda ») de l'anglais. Il s'agit d'une expression très répandue parmi les adolescentes brésiliens, ce qui expliquerait peut-être son choix pour le titre de l'ouvrage.
} 
Prenant en compte le traitement donné à ce sujet par Oliveira et Silva, Risso et Urbano (2006), ${ }^{10}$ nous avons réalisé une étude des MD présents dans l'ouvrage analysé. Nous y avons constaté plusieurs occurrences de MD, comme indique le tableau ci-dessous:

Tableau 1: Classement des marqueurs discursifs présents dans Tipo assim: adolescente.

\begin{tabular}{|c|c|c|c|}
\hline \multicolumn{2}{|c|}{ Type de marqueur discursif } & $\begin{array}{c}\text { Nombre } \\
\text { d'occurrences }\end{array}$ & $\mathbf{\%}$ \\
\hline \multicolumn{2}{|c|}{ Textuel } & 17 & 18,20 \\
\hline \multirow{4}{*}{$\begin{array}{c}\text { Marqueurs } \\
\text { Interactionnels }\end{array}$} & Olha & 5 & 5,37 \\
\cline { 2 - 4 } & Entende & 9 & 9,67 \\
\cline { 2 - 4 } & Aí & 10 & 10,75 \\
\cline { 2 - 4 } & Nossa & 11 & 11,82 \\
\cline { 2 - 4 } & Sabe & 14 & 15,15 \\
\hline Total & Né? & 27 & - \\
\hline
\end{tabular}

Comme on peut le vérifier sur le tableau, la plupart des MD sont du type interactionnel, ${ }^{11}$ fonctionnant donc comme des séquenceurs discursifs; voyons quelques exemples: ${ }^{12}$

(01) Foi tão natural, sabe? $\approx$ Ça a été si naturel, tu sais? ${ }^{13}$ (BOUER, FRANCINE, 2005: 9).

(02) Eaí, você já beijou? ₹ Et alors, t'as déjà embrassé quelqu'un? (BOUER, FRANCINE, 2005: 15).

(03) Há sempre um pouco de transgressão, né!? ₹ Il y a toujours un peu de transgression, n'est-ce pas!? (BOUER, FRANCINE, 2005: 16).

(04) Não para fofocar, só para saber mesmo, entende? $\approx$ Pas pour ragoter, juste pour savoir, tu sais? (BOUER, FRANCINE, 2005: 20).

Le tableau suivant, qui adopte le classement proposé par Guerra (2007) pour les fonctions exercées par les MD interactionnels, présente exclusivement les occurrences de ce type de marqueur dans l'ouvrage:

${ }^{10}$ Dans ce travail les auteurs présentent des critères nous permettant de distinguer des marqueurs discursifs interactionnels et textuels.

${ }^{11}$ Parmi les MD ayant des fonctions textuelles, nous trouvons des occurrences de « mas » et de « aí » comme séquenceurs topiques. Nous avons choisi de ne pas analyser ces cas, vu que leur occurrence dans l'ouvrage en question n'est pas significative.

${ }^{12}$ Dans les extraits, les modes d'expression mis en relief sont en italique.

${ }^{13}$ En mathématiques, le symbole $\approx$ signifie « est presque égal à » $\approx\{\backslash$ displaystyle $\backslash$ approx $\}$ $\simeq\{\backslash$ displaystyle $\backslash$ simeq $\}$ et permet de donner une valeur approchée d'un nombre. On 1'utilise ici pour indiquer ques les traductions, surtout celles de certains marqueurs discursifs, ont été faites par approximation, vu que, pour certains de ces marqueurs, il est assez difficile de trouver des correspondants en français. 
Tableau 2: Classement des marqueurs discursifs d'interaction repérés dans Tipo assim: adolescente

\begin{tabular}{|c|c|c|}
\hline $\begin{array}{c}\text { Fonction du marqueur } \\
\text { discursif interactionnel }\end{array}$ & Nombre d'occurrences & $\mathbf{\%}$ \\
\hline Injonctif & 5 & 6,5 \\
\hline Initiateur & 11 & 14,4 \\
\hline Checking & 60 & 79,1 \\
\hline Total & 76 & - \\
\hline
\end{tabular}

Comme nous pouvons le constater, la plupart des MD interactionnels exercent la fonction de checking (vérification). Ils expriment une orientation du sujet énonciateur en direction de l'énonciataire, en vue d'obtenir l'approbation du discours. Plus spécifiquement, comme le montre le premier tableau, le MD le plus fréquent est le marqueur de vérification né? (n'est-ce pas?) L'emploi de ce MD a pour objectif d'obtenir l'approbation par rapport au topique de l'interaction, ce qui met en évidence le rapport d'amitié qu'il y a entre les énonciateurs et contribue à la dilution de l'éloignement ou à l'atténuation de l'hiérarchie entre eux. Ainsi, la présence des MD d'interaction rapproche les énonciateurs et implique leur engagement quant à la continuité de l'intéraction. À ce propos, il faut dire que l'ouvrage se présente comme un dialogue entre les énonciateurs, qui se manifestent de manière informelle et proche, avec des récits d'expériences et de sentiments personnels; d'où la fréquence des MD donnant au texte un ton informel, comme dans un dialogue entre amis (et non pas entre les professionnels qui assument l'écriture du livre, soit un médecin et une présentatrice de télévision).

La fréquence des occurrences des MD interactionnels caractérise, ainsi, la scénographie du texte, qui peut être considérée comme celle d'une conversation informelle entre deux amis à propos de l'adolescence, une confidence entre personnes proches qui ont des problèmes en commun. Les garants du texte sont donc deux amis qui s'expriment informellement et d'une manière qui relève de l'intimité et de la proximité, d'où la présence significative et récurrente des marqueurs discursifs du type interactionnel, lesquels témoignent la nature interactive de la scénographie et la relation de proximité des énonciateurs. À cet égard, il est utile de rappeler que cette scène de dialogue, de confidences entre amis, n'est, en tant que scénographie, qu'une simulation soutenue par le propre discours, car il est clair qu'il ne s'agit pas de la transcription d'un dialogue à proprement parler, mais de sa simulation, basée sur une certaine image du public projeté. 


\subsection{Les éléments modaux}

Passons à l'analyse du second élément d'investigation du texte, la modalité, en mettant l'accent sur son expression lexicale. La modalité est une catégorie du langage qui désigne la relation de l'énonciateur avec l'énoncé. Plus exactement, la modalité reflète le jugement de l'énonciateur sur le contenu propositionnel de son énoncé aussi bien qu'elle révèle la relation intersubjective qui est instaurée par l'énonciation. ${ }^{14}$ Par conséquent, l'étude de la modalité peut contribuer effectivement pour l'analyse de l'image du sujet énonciateur dans la mesure où elle met en évidence la façon dont l'énonciateur se positionne par rapport à ce qu'il dit (s'il manifeste, par exemple, des doutes ou de la certitude quant à ce qu'il affirme) et le type de relation qu'il établit avec son énonciataire (par exemple, s'il s'adresse à celui-ci de manière autoritaire ou non).

Pour analyser cet aspect de la matérialité linguistique, nous avons fait, comme dans le cas précédent, une liste de toutes les occurrences des éléments lexicaux modalisateurs du texte. On peut voir le résultat de cette enquête, qui adopte le classement proposé par Hengeveld (2004), ${ }^{15}$ dans le tableau suivant:

Tableau 3: Classement des éléments lexicaux modaux présents dans l'œuvre Tipo assim: adolescente

\begin{tabular}{|c|c|c|}
\hline Type de Modalité & Nombre d'occurrences & $\mathbf{\%}$ \\
\hline Volitionnelle & 1 & 0,3 \\
\hline Facultative & 14 & 4,7 \\
\hline Évidentielle & 51 & 17,3 \\
\hline Déontique & 108 & 36,4 \\
\hline Épistémique & 122 & 41,3 \\
\hline Total & 296 & - \\
\hline
\end{tabular}

Comme le tableau le révèle, le nombre des occurrences des modaux épistémiques qui indiquent l'évaluation de possibilité ou de certitude de l'énonciateur quant à ce qu'il dit est relativement elevé; ainsi, nous pouvons remarquer que la manifestation de possibilité ou d'incertitude est récurrente. À ce propos, en observant les énoncés où les énonciateurs expriment le doute au moyen d'éléments lexicaux modaux, nous remarquons qu'il s'agit d'énoncés dans lesquels les énonciateurs décrivent une situation hypothétique relative à l'adolescent ou dans lesquels ils cherchent les motivations probables ayant amené ce jeune à adopter un certain comportement. Par exemple:

${ }^{14}$ Cf. SAINT-PIERRE (1992, apud NEVES, 1996).

${ }^{15}$ Dans ce travail, de nature fonctionnaliste, l'auteur classe les modalités à partir de deux critères principaux: le type de cible de l'évaluation, c'est-à-dire la partie de l'énoncé qui est modalisée, et le domaine sémantique à partir duquel l'évaluation est faite. Ainsi, par le paramètre cible de l'évaluation, il identifie les modalités orientées vers le participant, vers l'événement et vers la proposition, et par le paramètre du domaine sémantique, les modalités facultative, déontique, volitionnelle, épistémique et évidentielle. 
(05) Assim, pode ser que exista um turbilhão acontecendo dentro desse adolescente, mas a gente não consegue acessar. $\approx$ Comme ça, il peut y avoir un tourbillon en train de se produire à l'intérieur de cet adolescent, mais on ne peut pas y accéder. (BOUER, FRANCINE, 2005: 30).

(06) Mas também pode ocorrer de ele se sentir perdido mesmo, de não saber o que fazer e não querer pensar em nada. $\approx$ Mais il peut arriver aussi qu'il se sente perdu, qu'il ne sache pas quoi faire et qu'il ne veuille penser à rien. (BOUER, FRANCINE, 2005: 32).

On peut remarquer que les assertions sur l'adolescence sont accompagnées de plusieurs exemples, ce qui peut justifier la grande occurrence des modaux épistémiques. Les énonciateurs, en affirmant leurs « thèses » sur l'adolescence, utilisent plusieurs exemples pour illustrer les raisons sur lesquelles elles se basent. Ces exemples, à leur tour, représentent des situations possibles que peut vivre un adolescent, ce qui justifie la grande fréquence de ce type modal dans l'ouvrage. De cette manière, nous voyons que les énonciateurs ne manifestent pas d'incertitude quant aux idées qu'ils défendent, mais concernant les situations dans lesquelles les jeunes sont impliqués.

Nous notons aussi que les modaux épistémiques sont, en général, orientés vers l'événement, ce qui donne un caractère moins subjectif aux évaluations réalisées par les énonciateurs. ${ }^{16}$ Cela signifie qu'ils ne se compromettent pas avec ces évaluations, présentant la certitude ou le doute comme ne relevant pas de leur responsabilité.

Un autre aspect qui a attiré notre attention concerne la présence des marqueurs d'inférence, spécialement ceux relatifs à l'énonciateur (le verbe « achar»). Voyons quelques exemples:

(07) Então, acho que, talvez, o que mais tenha marcado a adolescência, na minha memória, seja essa divisão, esses dois lados da moeda. (BOUER, FRANCINE, 2005: 7-8). $\approx$ Donc, je crois que ce qui a peut-être le plus marqué l'adolescence, dans ma mémoire, c'est cette division, ces deux côtés de la médaille.

(08) Eu acho que isso é uma grande perda, uma coisa que tem consequências diversas, não ter que enfrentar limites. $\approx$ Je pense que ça, c'est une grande perte, une chose qui a plusieurs conséquences, que de ne pas avoir à affronter des limites. (BOUER, FRANCINE, 2005: 11).

Ces modaux indiquent un plus grand engagement des énonciateurs avec ce qu'ils disent, puisqu'ils se présentent comme la source de la connaissance des propositions. En outre, ces modaux impriment un degré d'incertitude considérable aux énoncés en indiquant que l'information qu'ils véhiculent dépend de la confiance accordée à l'énonciateur, source de l'évaluation. Ainsi, ces évidentiels provoquent une atténuation de l'assertivité des énoncés, car ils indiquent un doute et/ou une incertitude de la part de l'énonciateur, ce qui rend le discours

\footnotetext{
${ }^{16}$ Cf. Hengeveld (2004).
} 
plus personnel et moins assertif. En outre, comme il s'agit d'une manifestation de la subjectivité, ils renforcent le caractère personnel du discours et signalent la proximité qu'il y a entre les énonciateurs, ce qui renforce aussi la caractérisation de la scénographie comme un dialogue entre amis, entre personnes proches, qui échangent des informations et laissent transparaître leurs doutes.

Concernant les modaux facultatifs, la fréquence de leur emploi est relativement basse (4,7\%). Les énonciateurs ne proposent pas, par exemple, que les situations considérées comme problématiques dans lesquelles se trouvent les adolescents soient résolues par leur capacité de dépassement ni qu'ils auraient le pouvoir de changer leur propre realité, comme l'affirment les énonciateurs dans le cas des ouvrages donnant des conseils aux adultes. ${ }^{17}$ En général, l'utilisation des facultatifs a aussi un rapport avec les situations hypothétiques mentionnées par les énonciateurs, comme on le constate dans l'extrait ci-dessous:

(09) De um lado, eu posso tudo, vou em frente, alguma coisa nova está começando; e, do outro, um freio de mão que é, na verdade, seu mesmo [...]. (BOUER, FRANCINE, 2005: 8). ₹ D'un côté, je peux tout, je fonce, quelque chose de nouveau est en train de commencer; et de l'autre côté, un frein à main qui, en réalité, relève de moi-même.

D’autre part, on repère aussi des éléments lexicaux déontiques. En voici quelques exemples:

(10) Ao entrar na adolescência, a gente percebe que tem que romper com esses limites [...] (BOUER, FRANCINE, 2005: 41). $\approx$ En passant à l'adolescence, on s'aperçoit qu'il faut rompre avec ces limites.

(11) Para crescer, é necessário romper com muitas coisas. (BOUER, FRANCINE, 2005: 41). $\approx$ Pour grandir, il faut rompre avec beaucoup de choses.

(12) Você tem de ser capaz de reconhecer quando o pessoal está agindo mal [...] (BOUER, FRANCINE, 2005: 45). $\approx$ Il faut être capable de reconnaître quand les gens agissent mal $[\ldots]$

D’une manière générale, les éléments lexicaux modaux déontiques sont employés pour que l'énonciateur puisse imposer des volontés à l'énonciataire et contrôler son comportement en lui adressant des ordres, des autorisations ou des interdictions. Dans le texte, 58,3\% de ces modaux, soit 63 occurrences, sont orientés vers le participant; le restant, 45 occurrences (ou 41,7\% du total des occurrences d'éléments lexicaux déontiques), sont orientés vers l'événement.

${ }_{17}$ À ce propos, voir les résultats de Brunelli et Hattnher (2011) sur des ouvrages donnant des conseils aux adultes. 
Quand la modalisation déontique est orientée vers l'événement, l'obligation (ou l'autorisation, ou même l'interdiction) est représentée comme une règle générale de conduite, sans indication de sa source, ce qui atténue le caractère autoritaire propre à cette qualification modale. Quant aux modaux déontiques tournés vers le participant, bien qu'ils soient prédominants, nous constatons que, dans la plupart des cas, le rôle d'autorité de l'énonciateur se trouve atténué par le recours à une stratégie quelconque, ce qui est un indice de plus de la relation de proximité qu'un énonciateur maintient avec l'autre. Il y a donc toujours une atténuation des ordres et des interdictions par des recours qui affectent l'indication de la cible à laquelle se dirige la modalisation déontique orientée au participant.

Parmi les formes d'atténuation de la modalité déontique retrouvées dans l'ouvrage, il y a l'emploi du sujet indéterminé, les propositions sans sujet, l'emploi du pronom você à valeur impersonnelle, l'emploi d'expressions impersonnelles. Le pronom você ne concerne pas l'énonciataire immédiat de l'énonciateur (dans ce cas l'autre énonciateur, puisqu'il s'agit d'un dialogue entre deux amis), mais il renvoie à tous les énonciataires possibles; il s'agit, donc, du você impersonnel (cf. Fiorin, 2001) et son emploi aide à diminuer le ton autoritaire de l'énonciateur. Les expressions ayant trait à la $3^{\mathrm{e}}$ personne indiquent qui doit assumer certains devoirs et identifient ceux-ci, sans signaler directement l'énonciataire. À l'exception de ce dernier recours, toutes les autres formes mentionnées rendent générique la référence à la cible de l'évaluation modale, c'est-à-dire qu'on n'identifie pas de façon précise à quel(s) sujet(s) sont adressés les « ordres » ou les « interdictions » faits par les énonciateurs de l'ouvrage, ce qui diminue le ton injonctif auquel est associé ce type de modalité.

Ce phénomène de l'atténuation de la force modale déontique peut être considéré comme un indice que l'orientation de la part des énonciateurs est faite d'une manière moins autoritaire, plus intime, dans laquelle on ne relève pas l'établissement d'une hiérarchie bien définie entre les personnes impliquées et dans laquelle l'énonciateur prend une posture moins impérative par rapport à son énonciataire (que ce soit l'autre énonciateur ou l'adolescent qui le lit). Ainsi, les énonciateurs ne se présentent pas comme des autorités à qui on doit obéir sans restriction, mais comme ceux dont l'expérience leur permet de conseiller et de suggérer. Leurs orientations ont plutôt la forme de suggestions ou de descriptions de conduites naturelles, c'est-à-dire que leurs orientations se présentent plus comme des conseils que comme des ordres ou des interdictions.

Une autre forme linguistique pouvant marquer la modalisation déontique d'un énoncé a trait à l'emploi des formes impératives du verbe. Elles représentent de façon plus marquée le caractère autoritaire de l'énonciateur et, par conséquent, la position hiérarchiquement supérieure de celui-ci par rapport à l'énonciataire. Dans l'ouvrage, on peut constater que la fréquence des impératifs est relativement basse (27 ocurrences) et leurs contextes d'utilisation, dans la plupart des cas, sont la reproduction des propos des parents à leurs enfants lorsqu'ils assument leur autorité sur ces derniers, ou des recommandations et des ordres qu'ont entendus les adolescents. Nous citons les extraits suivants: 
(13) E a mãe da menina também estava na minha casa e também disse algo assim: "Venham já para a casa os dois, porque vocês andaram aprontando". (BOUER, FRANCINE, 2005: 12). $\approx$ Et la mère de la fille était elle-aussi chez moi et a dit elle-aussi quelque chose comme: «Rentrez sur-le-champ tous les deux, car vous avez certainement fait des bêtises ».

(14) Muitas vezes, os pais, a mídia ficam tentando passar aquela mensagem: "Não faça as outras coisas só porque os outros fazem, não se deixe levar pela influência do grupo" - principalmente na questão de drogas. "Seja independente, não faça alguma coisa só porque todo mundo faz, só pela pressão do grupo". (BOUER, FRANCINE, $2005: 41)$. $\approx$ Souvent les parents, les médias essaient de faire passer ce message: «Ne fais pas les choses parce que les gens les font, ne te laisse pas entraîner par l'emprise du groupe » - surtout en ce qui concerne les drogues. "Sois indépendant, ne fais pas quelque chose seulement parce que tout le monde le fait, seulement par la pression du groupe ».

On remarque une distance de la part des énonciateurs par rapport à ces voix (que ce soit les ordres des parents ou celles que les adolescents reçoivent d'autrui), soit ils n'assument pas cette autorité, ils se montrent plus flexibles et moins affirmatifs dans leurs thèses.

\subsection{Le lexique employé}

Passons à présent au dernier aspect sélectionné pour cette analyse : le lexique. Nous observons l'occurrence de termes et d'expressions informels, qui pourraient caractériser aussi le langage des adultes, mais qui sont employés fondamentalement par les jeunes, ce qui rapproche les énonciateurs de l'énonciataire projeté par le discours, quoique les expressions utilisées se rapportent à un langage des jeunes non contemporain mais relatif à l'adolescence des énonciateurs. Ainsi, on pourrait dire qu'il s'agit plus exactement d'un simulacre d'adolescence relevant de la perspective des énonciateurs. Nous présentons ci-après quelques extraits de ce lexique:

(15) E qual era a grande nóia da minha mãe? $\approx$ Et quelle était la grande paranoïa de ma mère? (BOUER, FRANCINE, 2005: 20).

(16) Agora, Jairo, fala sério: O que é a escola? $\approx$ Maintenant Jairo, franchement, qu'est-ce que c'est que l'école? (BOUER, FRANCINE, 2005: 33).

(17) Olha que viagem, né? $\approx$ Regarde, quel délire! N'est-ce pas? (BOUER, FRANCINE, 2005: 69).

Nous constatons aussi que le lexique employé, bien qu'il soit informel et constitué de mots d'argot, ne présente pas de termes considérés tabous. Le vocabulaire ne relève pas d'un langage "vulgaire ». Les énonciateurs sont donc des jeunes qui s'expriment d'une manière assez informelle, propre à ce segment social, mais qui ne sont pas des « rebelles sans cause » qui brisent les règles de comportement, qui choquent par leurs attitudes et, dans ce cas précis, par leur manière de s'exprimer (attitude verbale). À cette rupture des règles de 
comportement serait lié aussi le manque de respect aux règles plus rigides du langage soutenu (comme enfreindre les règles d'accord verbal et nominal, particulièrement), ce qu'on n'observe pas dans l'ouvrage en question, c'est-àdire qu'il ne s'agit vraiment pas d'un adolescent voulant briser les conventions. Ainsi, le parler informel et juvénile des énonciateurs se limite à ce qui est toujours considéré comme acceptable selon certains critères du langage.

Si l'on considère ces résultats dans leur ensemble, on peut dire que la scène d'énonciation où sont insérés les garants du discours, soit la scénographie, est vraiment celle d'un dialogue où il y a un échange d'expériences entre les énonciateurs. Ils n'énoncent pas comme deux spécialistes de l'adolescence qui parlent du sujet en se basant sur leurs points de vue spécifiques (celui d'un médecin, par exemple), mais comme deux amis qui réfléchissent à l'adolescence en fonction de leurs expériences et de leurs observations quotidiennes; par conséquent leur énonciation est informelle et relate les faits qui marquent la vie des jeunes. Il ne s'agit pas non plus d'un dialogue entre deux énonciateurs convaincus et sûrs d'eux-mêmes, sûr de leurs convictions, centrés sur eux-mêmes, mais de deux amis, deux personnes proches et suffisamment intimes pour partager entre eux des faits personnels, dans une relation sans asymétrie entre eux (mais aussi entre eux et le public cible de l'ouvrage, soit les adolescents), comme lors d'une confidence.

Après cette exposition, on peut dire que l'image de l'adolescent en question s'éloigne de certains stéréotypes (négatifs ?): il ne s'agit pas d'un transgresseur ou d'un réformateur des règles sociales et des mœurs, ce n'est pas un « rebelle sans cause »; c'est un être qui a des doutes, des craintes, manquant d'assurance, qui vit un processus d'individualisation et d'autonomie.

\section{ORIENTATIONS SEXUELLES POUR LE JEUNE «ÉCERVELÉ »}

Les autres textes que nous analyserons s'appellent Caderneta de Saúde do Adolescente (Carnet de santé de l'adolescent, cité dans les exemples comme C1) et Caderneta de Saúde da Adolescente (Carnet de santé de l'adolescente, cité dans les exemples comme C2), qui font partie d'un ensemble de textes de genres divers (livrets, manuels, campagnes publicitaires), destinés aux enseignants, aux professionnels de la santé et au public en général (jeunes et adultes), produits par le gouvernement fédéral brésilien depuis la fin de la décennie 1990 et dont l'objectif était d'instruire les individus sur les pratiques sexuelles sûres pour eux-mêmes et pour les autres membres de la collectivité. Ce point de repère temporel se justifie dans la mesure où la reconnaissance de la relation indissociable entre les pratiques sexuelles des individus et le développement social s'est effectuée à une échelle mondiale en 1994, avec la définition, par l'ONU, des Droits sexuels et reproductifs. Ainsi, cet événement (au sens de Foucault) ${ }^{18}$ a eu des effets très significatifs qui ont défini les orientations des politiques publiques nationales en matière d'éducation sexuelle, de sorte qu'une vaste production textuelle a été mise en circulation dans l'objectif d'instruire les gens quant à ce que doit être une « conduite sexuelle saine».

\footnotetext{
${ }^{18}$ Cf. Foucault (2000).
} 
Dans ce contexte, on trouve les carnets cités, qui sont des productions destinées spécifiquement à un public jeune. Plus exactement, ce sont une sorte de journaux intimes destinés aux filles et aux garçons agés entre 10 et 19 ans. Ils peuvent y noter des données personnelles comme leur adresse, leur âge, etc., ainsi que les vaccins, les allergies ou les opérations chirurgicales subies. Outre ces aspects liés à la santé de l'adolescent, ces carnets comportent de nombreux conseils sur l'alimentation, la santé buccale, les boutons d'acné et l'hygiène, sur la sexualité et les méthodes contraceptives, et des orientations indiquant comment procéder en cas de violence (y compris sexuelle) ou de grossesse.

Chacun des deux contient 50 pages, et une grande partie de leurs contenus est identique. Ce qui les différencie ce sont les informations sur l'anatomie et celles concernant la grossesse : tandis qu'aux filles on donne des informations sur leur droit à une assistance prénatale et lors de l'accouchement et après, et sur le fait que l'école est obligée par la loi d'offrir à la mère adolescente la possibilité de passer ses examens à une date ultérieure dès lors qu'elle présente un justificatif d'absence, etc., aux garçons on signale qu'ils doivent assumer la paternité et ne pas abandonner leurs études.

La définition du public cible a des implications importantes pour l'ethos du garant du discours qui émerge des carnets. Les stratégies sont dans une certaine mesure les mêmes. La mise en page des deux carnets est colorée, avec des polices de plusieurs types, et ils sont pleins d'illustrations et de photos de garçons et de filles de différentes ethnies (des noirs, des blancs, des indiens et des orientaux), en plus de ceux en fauteuil roulant et des handicapés visuels. On s'adresse à l'énonciataire par você - dans une simulation de dialogue avec le jeune et non plus entre deux énonciateurs -, et le lexique et les structures des phrases font émerger un ethos juvénil, en même temps que l'image du jeune est celle d'un individu plein de doutes, ayant donc besoin d'une voix ferme, mais solidaire, l'incitant à faire les bons choix. Voyons quelques extraits de ces carnets:

(18) Espinhas? Calma, isso não é o fim do mundo! Uma boa dica: lavar o rosto 2 a 3 vezes ao dia com sabonete esfoliante pode ajudar no controle da oleosidade. Se o problema incomodar, procure um serviço de saúde, pois existe tratamento, viu? Sem drama! $\approx$ Des boutons d'acné? Du calme, ce n'est pas la fin du monde! Un petit conseil: se laver le visage 2 ou 3 fois par jour avec une savonnette exfoliante, ça peut aider à éviter la peau grasse. Si le problème persiste, va chercher un centre de santé, car des traitements existent, ok? Pas de panique! (C1, p. 32; C2, p. 32)

(19) A primeira relação sexual, para ser segura e prazerosa, só deve ocorrer a partir do momento em que você se sentir preparada. Essa escolha é sua! Respeite seu próprio ritmo e não se deixe levar pelas pressões dos outros. $\approx$ Pour que la première relation sexuelle soit sûre et agréable, elle ne doit avoir lieu qu'à partir du moment où tu te sens prête. C'est à toi de choisir! Respecte ton propre rythme et ne te laisse pas influencer par les pressions sociales. (C2, p. 42) 
(20) A partir de estímulos hormonais, podem ocorrer também mudanças no tom de voz. Inicia-se o aumento dos testículos e do pênis. Também há o aparecimento de pelos na região pubiana, nas axilas, no rosto e no restante do corpo. E não para por aí não! É comum o aumento da transpiração e odores (cheiros), ${ }^{19}$ principalmente nas axilas (o popular "CC") ${ }^{20}$ e nos pés (o "chulé"). ${ }^{21}$ Por tudo isso o banho diário é muito importante! $\approx$ Les stimulations hormonales peuvent entraîner aussi des changements du timbre de la voix. Cela s'initie par une augmentation des testicules et du pénis. Il y a aussi des poils qui apparaissent sur la région pubienne, sous les aisselles, sur le visage et sur tout le reste du corps. Et ça ne s'arrête pas là! Souvent il y a une augmentation de la transpiration et des odeurs, surtout aux aisselles et aux pieds. Pour toutes ces raisons, il est très important de se doucher tous les jours. (C1, p. 31)

(21) É normal que seu pênis apresente uma secreção branca e espessa, que também tem um outro nome "dificil": chama-se esmegma, na glande (a cabeça do pênis, lembra?), sob o prepúcio. É muito importante você limpá-la todos os dias no banho com água e sabão. Para isso, primeiro puxe o prepúcio deixando aparecer toda a glande para uma boa higiene. Se você não conseguir puxar totalmente a pele para descobrir a glande, sentir dor ou apresentar feridas ou secreções, procure o serviço de saúde para saber o que está acontecendo, combinado? $\approx \mathrm{Il}$ est normal que ton pénis présente une sécrétion blanche et épaisse, qui a aussi un autre nom "difficile »: cela s'appelle le smegma, sur le gland (la tête du pénis, tu t'en souviens?), sous le prépuce. Il est très important de la nettoyer tous les jours quand tu te douches, avec de l'eau et du savon. Pour cela, d'abord tire le prépuce afin de laisser apparaître tout le gland pour une bonne hygiène. Si tu n'arrives pas à tirer complètement la peau pour découvrir le gland, que ça fait mal ou qu'il y a des blessures ou des sécrétions, adresse-toi à un centre de santé pour savoir ce qui se passe, d'accord? (C1, p. 38)

Dans ces extraits, on peut observer qu'un ton professoral est assumé par l'énonciateur - un enseignant «sympa » qui sait parler aux jeunes, qui connait leurs attentes et leurs doutes. Cet énonciateur simule une conversation « tête à tête » avec les adolescents, mais dans celle-ci il y a une dénivellation des positions occupées par l'énonciateur et par l'énonciataire. L'énonciateur est un adulte qui informe et soutient les jeunes qui traversent une période de doutes et de découvertes. On le voit, par exemple, aux instructions données sur l'hygiène et aux conseils pour résoudre le problème des boutons. En outre, il y a des marqueurs discursifs interactionnels, des explications sur les termes « difficiles » (smegma, gland), l'utilisation d'un lexique typiquement juvénile, ce qui fait ressortir l'image d'un adulte qui joue le rôle d'un tuteur qui s'adresse aux jeunes d'une manière qui leur est familière (emploi de marqueurs discursifs interactionnels ; emploi d'un lexique juvénile) et qui cherche à leur faciliter la compréhension des carnets (d'où l'explication des termes « difficiles »).

${ }^{19}$ Le portugais a deux mots pour traduire le mot français « odeur » : odor, plus soutenu, et cheiro plus courant. C'est pour cela qu'après avoir utilisé le pluriel odores l'auteur donne son correspondant plus courant (cheiros), en guise d'explication, ce que n'est pas possible en français.

${ }^{20}$ O popular " $C C$ ”' (le populaire « CC ») n'a pas de traduction en français. Il s'agit d'une façon très populaire de parler de l'odeur qu'on a parfois sous les aisselles après avoir beaucoup transpiré. Quand ça arrive, on a l'habitude de dire que la personne a le CC.

${ }^{21}$ Le chulé, qui n'a pas de traduction en français, est un mot populaire pour parler de la forte odeur des pieds quand ils transpirent beaucoup. 
Nous observons aussi que la sexualité est présentée comme un droit et une source de plaisir et de bonheur - pourvu que l'on ait une attitude responsable lors des pratiques sexuelles. L'accent est donc mis sur le fait qu'une vie sexuelle adéquate exige un comportement responsable et que des « attitudes inadéquates »- le sexe sans protection - ont des conséquences graves pour la vie des jeunes. Bien que ce soit une information présente dans pratiquement tous les matériels éducatifs destinés à la population en général, ${ }^{22}$ le ton des textes adressés à un public de jeunes et d'adolescents est plus ferme - c'est-à-dire qu'on parle à des jeunes prétendument peu conscients et peu responsables, comme un adulte qui essaie de montrer l'importance d'avoir des responsabilités et de faire les bons choix. Il y a, d'une certaine manière, la reproduction de la disparité existante dans les relations entre jeunes et adultes, comme on le voit bien ici:

(22) É preciso cuidar da sua saúde física, mental e emocional e se preparar para assumir as responsabilidades e consequências que fazem parte da sua escolha, com conhecimentos e recursos que possam ajudá-la/o a se prevenir de uma gravidez não planejada e a se proteger de doenças, como as sexualmente transmissíveis - DST/Aids. $\mathrm{O}$ uso do preservativo só depende de você. Essa é a única forma de garantir sua segurança. $\approx$ Il faut prendre soin de sa santé physique, mentale et émotionnelle, et se préparer à assumer les responsabilités et les conséquences qui découlent de son choix, avec des connaissances et des ressources qui peuvent aider à prévenir une grossesse non désirée et à se protéger contre les maladies, comme celles sexuellement transmissibles - MST/SIDA. L'usage du préservatif ne dépend que de toi. C'est la seule façon d'assurer ta sécurité. (C1, p. 44; C2, p. 44)

(23) Por último, lembre-se: Você é a dona/o da sua vida e, por isso mesmo, pode participar da construção de um mundo melhor. Um mundo sem preconceitos, sem discriminação. Um mundo mais pacífico, mais solidário e menos violento. [...] Por isso, escolha bem as sementes que vão gerar os frutos que você deseja colher. $\approx$ Pour finir, rappelle-toi: Tu es le maître/la maîtresse de ta vie et, grâce à cela, tu peux participer à la construction d'un monde meilleur. Un monde sans préjugés, sans discrimination. Un monde plus pacifique, plus solidaire et moins violent. [...] Donc, choisis bien les graines qui vont produire les fruits que tu désires récolter. (C1, p. 49; C2, p. 49)

Ainsi, l'image du jeune qui émerge des deux carnets est celle d'un jeune qui a besoin d'orientation, plus exactement celle d'un adolescent un peu « écervelé » qui n'a pas la juste notion des conséquences de l'acte sexuel et qui a donc constamment besoin d'orientation et, parfois, même d'être « averti ». Mais d'autre part, cet « écervellement », si l'on peut dire, n'est jamais abordé d'un point de vue moral. Le ton d'avertissement, qui parfois devient un peu plus menaçant, a toujours pour objectif de rendre les jeunes conscients pour qu'ils puissent assumer de façon autonome la direction de leurs vies.

${ }^{22}$ Pour les résultats d'analyses faites sur des matériels d'éducation sexuelle tournés vers d'autres publics, voir Fossey (2011). 


\section{CONSIDÉRATIONS FINALES}

D'après les analyses présentées, on peut dire que l'image de l'adolescent qui se trouve dans le discours d'orientation pour les adolescents ne se rapproche pas de certaines images propagées par le sens commun sur l'adolescence (lesquelles soulignent l'aspect de révolte et de rébellion de ces jeunes ou qui les caractérisent comme des gens qui dérangent ou ennuient les autres, particulièrement leurs parents ou les personnes plus âgées $)^{23}$ et garde des points communs avec ce que dit le discours de la psychologie sur cette phase. Selon des auteurs s'inscrivant dans le cadre théorique de la psychologie du développement (cf. JERSILD, 1961), l'adolescent s'insère dans une période de la vie marquée par la recherche d'identité ou d'une personnalité propre, un processus résultant de sa maturation biologique, sociale, morale, intellectuelle, vocationnelle et émotionnelle. Les études de ce courant théorique mentionnent aussi l'angoisse et le manque d'assurance qui caractérisent les sentiments des adolescents, résultat de ce processus de construction identitaire et qui s'ajoutent au gain d'autonomie et de responsabilité inhérent à cette phase.

L'adolescent brésilien des textes analysés, comme on le vérifie dans ces analyses, n'est pas " un rebelle sans cause », un individu qui brise les règles de comportement et crée ses propres normes, ni quelqu'un qui cause des ennuis à ses proches. Il s'agit d'un jeune immature et peu sûr de lui-même, parfois considéré comme un peu écervelé. Quoi qu'il en soit, il s'agit toujours de quelqu'un ayant besoin d'une orientation, que ce soit une explication sur le sexe ou sur la nécessité d'adopter une pratique d'hygiène, ou un avertissement sur les risques des pratiques sexuelles sans protection, ou encore un conseil concernant son comportement.

La manière d'énoncer des énonciateurs contribue à la construction de l'image du public auquel le discours s'adresse, ce qui se doit surtout aux marques d'informalité et d'interaction, propres aux scénographies où il y a une relation de proximité entre l'énonciateur et l'énonciataire. Même s'il y a une certaine différence entre le ton des carnets d'éducation sexuelle (plus ferme, révélant une certaine asymétrie entre l'énonciateur et l'énonciataire) et celui de l'ouvrage pour les adolescents (moins impérieux, sans présupposer cette relation d'asymétrie, que ce soit avec l'autre énonciateur ou avec le public auquel s'adresse le discours), les scénographies engendrées par les deux discours d'orientation sont très proches; il s'agit essentiellement d'un dialogue ${ }^{24}$ entre des énonciateurs qui entretiennent une certaine relation de proximité (d'où l'informalité et les marques d'interaction qui caractérisent le discours analysé).

${ }^{23}$ À ce propos, il faut noter qu'au Brésil cette image est si forte qu'elle a entraîné l'émergence d'un néologisme qui sert à caractériser les adolescents comme des gens importuns. Il s'agit d'un jeu de mots constitué à partir de la jonction du verbe aborrecer (ennuyer, embêter, emmerder) et du nom adolescente (adolescent), ce qui donne le mot aborrescente.

${ }^{24}$ Plus exactement, d'après ce que les analyses ont démontré, dans le cas des carnets il s'agit de la simulation d'un dialogue entre l'énonciateur et l'énonciataire ; quant au second cas, il s'agit d'un dialogue entre énonciateurs. 
Ainsi, quand la thématique est plus sérieuse et plus spécifique (santé, sexe et les risques que celui-ci implique), le ton plus ferme fait que les orientations transmises dans cette simulation de dialogue gagnent les contours d'un avertissement; par contre, quand il y a une gamme plus large et diversifiée de sujets, le ton moins impérieux caractérise la scénographie du dialogue, ce qui lui donne le caractère d'une confidence, et les orientations sont donc présentées comme des conseils, des suggestions.

Malgré cette différence, nous remarquons que, quand il s'agit de donner des orientations aux jeunes, l'image de l'énonciataire semble l'emporter sur celle de l'énonciateur et fonctionne, dans ces termes, comme la condition principale de production du discours, ce qui semble justifier l'ethos juvénile caractéristique de ce type de discours.

\section{$\overline{\text { RÉFÉRENCES }}$}

AMOSSY, R.; PIERROT, A. H. Stéréotypes et clichés. Paris: Editions Nathan, 1997 (version consultée: Estereótipos y clichés. Buenos Aires: Eudeba, 2001).

BOUER, J.; FRANCINE, S. Tipo assim: adolescente. Campinas: Papirus, 2005.

BRUNELLI, A. F.; DALL' AGLIO-HATTNHER, M. M. As qualificações do saber, do dever e do poder : uma análise linguística do discurso de autoajuda. In: BARONAS, R. G. (org.). Análise do discurso: teorizações e métodos. Campinas: Mercado de Letras, 2010, pp. 13-31.

BRÉSIL. Ministère de la Santé. Carnet de santé de l'adolescent. Brasilia: Ministère de la Santé, 2009. Disponible sur: http://bvsms.saude.gov.br/bvs/publicacoes/caderneta_saude_adolescente_menino.pdf Consulté le 7.07.2010.

BRÉSIL. Ministère de la Santé. Carnet de santé de l'adolescent. Brasilia: Ministère de la Santé, 2008. Disponible sur: http://bvsms.saude.gov.br/bvs/publicacoes/caderneta_saude_adolescente_menina.pdf Consulté le 7.07.2010.

FIORIN, J. S. As astúcias da enunciação: as categorias de pessoa, espaço e tempo. 2. ed. São Paulo: Ática, 2001, pp. 127-256.

FOUCAULT, M. Revenir à l'Histoire. In: Dits et écrits (1954-1988), tome II: 1976-1988. Paris: Gallimard, 1994 (version consultée: Retornar à história. In: MOTA, M. B. (org.). Michel Foucault - Arqueologia das ciências e história dos sistemas de pensamento. Coleção Ditos e Escritos, II. Trad. de Elisa Monteiro. Rio de Janeiro: Forense Universitária, 2000, pp. 282-295).

FOSSEY, M. Polêmica sobre sexo saudável: uma abordagem discursiva. 2011.182f. Tese (Doutorado em Linguística) - Instituto de Estudos da Linguagem, Universidade Estadual de Campinas, 2011.

FURLAN, M. M. Cenas de enunciação e ethos discursivo: análise do discurso de autoajuda para adolescentes. 2013. 162f. Dissertação (Mestrado em Estudos Linguísticos) - Instituto de Biociências, Letras e Ciências Exatas, Universidade Estadual Paulista Júlio de Mesquita Filho, São José do Rio Preto, 2013. 
GUERRA, A. R. Funções textual-interativas dos marcadores discursivos. 2007. 233f. Dissertação (Mestrado em Estudos Linguísticos) - Instituto de Biociências, Letras e Ciências Exatas, Universidade Estadual Paulista Júlio de Mesquita Filho, São José do Rio Preto, 2007.

HENGEVELD, K. Mood and modality. In: BOOIJ, G., LEHMANN, C., MUGDAN, J. (ed.). Morphology: a handbook on inflection and word formation. v. 2. Berlim : Mouton de Gruyter, 2004, pp. 1190-1202.

JERSILD, A. T. The psychology of adolescence. New York : Macmillan, 1957 (Version consultée: Psicologia da adolescência. Trad. de José Severo de Camargo Pereira e João Alves dos Santos. 2. ed. São Paulo: Nacional, 1961.)

MAINGUENEAU, D. Nouvelles tendances en analyse du discours. Paris: Hachette, 1987 (Version consultée : Novas tendências em Análise do Discurso. Trad. de Freda Indursky. Campinas: Pontes/Editora da UNICAMP, 1989).

MAIngueneAU, D. Cenas da enunciação. POSSENTI, S., SOUZA-E-SILVA, M. C. P. (org.). Divers traducteurs. Curitiba : Criar Edições, 2006.

MAINGUENEAU, D. A propósito do ethos. In: MOTTA, A. R.; SALGADO, L. (org.). São Paulo: Contexto, 2008, pp. 11-29.

MAINGUENEAU, D. Discours et analyse du discours - Introduction. Paris: Éditions Armand Colin, 2014 (Version consultée: Discurso e Análise do Discurso. Trad. de Sírio Possenti. São Paulo: Parábola, 2015).

NEVES, M. H. M. A modalidade. In: KOCH, I. G. V. (org.). Gramática do português falado. Campinas : Editora da UNICAMP/FAPESP, volume IV, 1996, pp.163-199.

OLIVEIRA E SILVA, G. M.; RISSO, M. S.; URBANO, H. Traços definidores dos marcadores discursivos. In: JUBRAN, C. C. A. S.; KOCH, I. G. V. (org.) Gramática do português culto falado no Brasil. Campinas: Editora da UNICAMP, 2006, pp. 403-425. 